\title{
Caractérisation expérimentale et numérique d'assemblages rivetés ferroviaires sous sollicitations quasi-statiques
}

\author{
Laurent Cébulski $^{1, a}$, Isabelle Guilloteau ${ }^{1}$, Pascal Drazetic ${ }^{2}$ et Éric Markiewicz ${ }^{2}$ \\ 1 ALSTOM TRAnSPORT, Parc d'Activités Lavoisier, BP 45, 59494 Petite Fôret, France \\ 2 LAMIH/LGM, Université de Valenciennes, Le Mont Houy, 59313 Valenciennes Cedex 9, France
}

Reçu le 25 novembre 2003, accepté le 3 septembre 2004

\begin{abstract}
Résumé - Les constructeurs ferroviaires utilisent de plus en plus le procédé d'assemblage par rivetage pour les avantages qu'il procure. Il conserve l'intégrité des matériaux contrairement au soudage et permet de rendre les structures plus modulaires. Par ailleurs, afin d'améliorer la sécurité dans les transports guidés, des critères de dimensionnement à la collision sont intégrés dans les phases de conception. Il apparaît dès lors nécessaire de mieux connaître le comportement de ces assemblages, le dimensionnement actuel étant gouverné par des règles empiriques. Pour les chocs à faible vitesse (accostages), la sollicitation peut être considérée quasi-statique en première approximation. La rationalisation du nombre, de la dimension et de la répartition géométrique des rivets est également un objectif prioritaire pour optimiser le dimensionnement des structures rivetées et conserver ainsi l'intérêt de ce procédé d'assemblage. Nous proposons de mettre en place une méthodologie fondée sur la simulation numérique du comportement local des rivets, afin d'en extraire les informations nécessaires à la mise en données d'une liaison macro-géométrique et d'un modèle macroscopique de rupture associés dans les modèles globaux de simulation. La méthodologie est ensuite vérifiée par un essai sur un sous-ensemble de structure industrielle.
\end{abstract}

Mots clés : Assemblages rivetés / rivets aveugles / tenue mécanique / éléments finis / essais arcan

\begin{abstract}
Numerical and experimental characterisation of railway riveted assemblies under quasi-static loadings. Railway manufacturers use extensively the riveting process with blind rivets for its advantages. It does not change the material properties (unlike welding) and allow to make more modular structures. Moreover, to improve safety in guided vehicles, some crash dimensionning criteria are now integrated in the design phase. It appears therefore necessary to better understand the behavior of these assemblies, which are dimensionned with empiric rules. In case of low speed shocks (like coupling), the loading can be considered as quasi-static as a first approximation. The rationalization of number, dimension and geometric distribution of the rivets is also a priority to optimize the design of riveted structures in order to preserve the interest of this assembly process. We propose a methodology based on numerical simulation of the rivetlocal behaviour, in order to extract the necessary information to put into data a macro-geometric link and its associated macroscopic rupture criterion, useful in global numerical models. After that, the methodology is verified with the test of an industrial structure part.
\end{abstract}

Key words: Riveted assemblies / blind rivets / mechanical behaviour / finite elements / arcan tests

\section{Introduction}

Traditionnellement, les structures ferroviaires sont essentiellement assemblées par soudage continu. Ces structures sont aujourd'hui principalement composées d'alliages d'aluminium (sauf pour les organes de traction et de choc). L'avantage est un gain de masse conséquent par

a Auteur correspondant :

laurent.cebulski@transport.alstom.com rapport à l'acier, mais la limite élastique demeure cependant beaucoup plus faible.

Le soudage accentue cet inconvénient en diminuant de moitié la limite élastique du matériau dans la zone affectée thermiquement, ce qui engendre des problèmes de tenue mécanique de la structure là où se situent les concentrations de contrainte (coins de portes, de baies...).

Face à ce problème mais aussi à la nécessité constante de réduire les coûts de fabrication, les constructeurs utilisent de plus en plus le procédé d'assemblage par rivetage 
qui permet également de rendre les structures plus modulaires. En effet, ce procédé offre la possibilité de modifier sensiblement la gamme de fabrication en concevant et en fabriquant des sous-ensembles entièrement équipés, l'assemblage étant effectué seulement en phase finale.

Par ailleurs, afin d'améliorer la sécurité dans les transports guidés, les constructeurs ferroviaires intègrent dans les phases de conception des critères de dimensionnement à la collision, critères qui varient selon les cahiers des charges des matériels concernés. Il apparaît dès lors nécessaire de mieux connaître le comportement des assemblages rivetés sous ce type de sollicitation.

Généralement, dans les modèles de simulation, les rivets sont représentés par des éléments équivalents de type macro-géométrique couplés à un critère de rupture en effort. La modélisation tient alors compte de l'agencement des pièces et le contact entre elles doit être géré pour éviter d'éventuelles interpénétrations au cours de la simulation.

Cependant, les confrontations calculs/essais les plus récentes montrent que la principale source de dispersion des résultats se situe au niveau de la mauvaise prédiction du comportement mécanique et de la rupture des assemblages. Les raisons sont multiples, telles une mauvaise connaissance du comportement mécanique non-linéaire des assemblages, du comportement des matériaux constituants, une trop grande simplicité des modèles macrogéométriques de représentation des liaisons, un critère de rupture mal caractérisé ou pas adapté, etc.

Le manque de connaissances singulier du comportement des rivets unitaires, et a fortiori des assemblages multi-rivets et multi-matériaux, nous a conduit à nous intéresser à une caractérisation la plus fidèle possible de la liaison rivet en termes de tenue mécanique et de modes de rupture, utilisable dans les simulations quasi-statiques (dans le cas de chocs à basse vitesse comme les accostages, les sollicitations sont considérées comme quasi-statique en première approximation) et dynamiques rapides des structures ferroviaires.

\section{Caractérisation d'un rivet ferroviaire}

\subsection{Présentation du type de rivet utilisé}

Les rivets utilisés chez le constructeur ferroviaire ALSTOM TRANSPORT sont des rivets aveugles HUCK BOM [1]. Outre leur haute résistance en traction et cisaillement, ces rivets présentent l'avantage de pouvoir assembler deux pièces dont l'une d'elles ne dispose pas d'accès par l'opérateur (côté « aveugle»). La figure 1 présente le rivet utilisé par le constructeur ferroviaire.

Le tableau 1 présente la séquence de mise en place de ce type de rivet.

\subsection{Problématique}

Une structure ferroviaire ne peut être discrétisée finement par des éléments solides pour des raisons évidentes

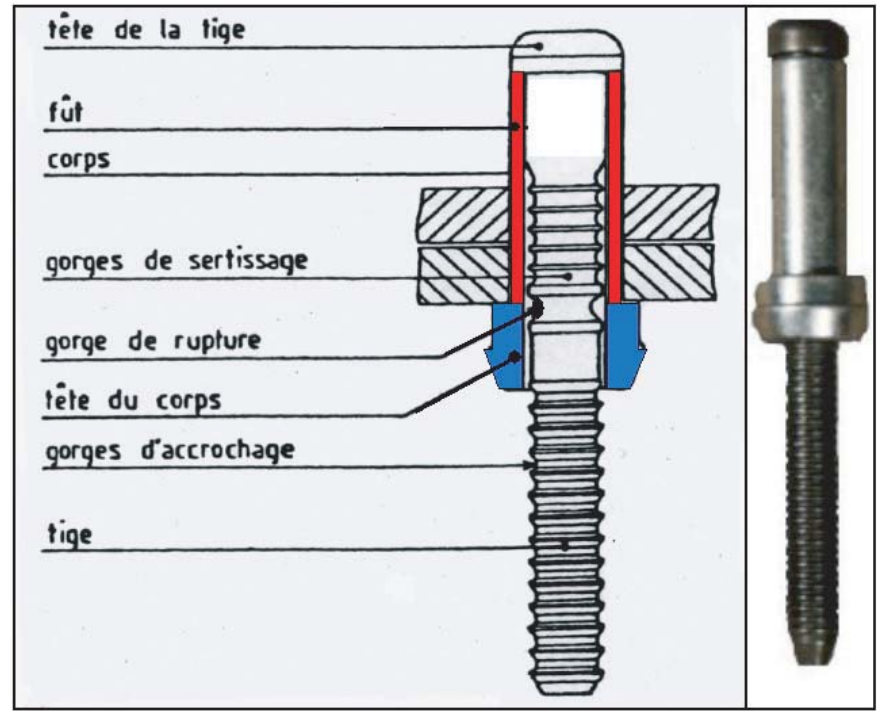

Fig. 1. Vue en coupe des composants d'un rivet.

de coût de calcul. Les assemblages sont modélisés par des éléments simplifiés nécessitant de caractériser la liaison concernée. Cependant, le choix du modèle simplifié et la sélection d'une méthode visant à accéder à une caractérisation la plus fidèle possible de ce modèle, constituent une difficulté non négligeable. Les techniques les plus fréquemment retenues consistent à opter pour un modèle de liaison ressemblant à un élément simple, censé reproduire le comportement d'un rivet formé. Le degré de représentativité requis peut inclure les comportements non-linéaires et la rupture. Il demeure toutefois des limites, comme la prise en compte de l'endommagement des tôles, ou encore des effets géométriques liés à la présence de perforations.

En effet, lorsqu'un assemblage riveté est sollicité avec ce type de rivets, et du fait de la haute résistance de ces derniers, on constate à la fois un endommagement du rivet concerné mais aussi des plaques assemblées (phénomène de matage). De plus, à chaque configuration d'assemblage correspond une combinaison (réponse globale effort/déplacement, mode de rupture) qui lui est propre. Le changement d'un seul paramètre de l'assemblage (matériaux ou épaisseur des plaques, couplage, diamètre et plage de serrage du rivet) a une influence sur son comportement et sa tenue mécanique.

Deux normes proposent des modèles simplifiés de calcul des assemblages rivetés :

- Dans la norme anglaise (British Standard) [2] figurent les valeurs de résistance des rivets acier et aluminium sous diverses sollicitations, dans le cas du serrage de plaques en aluminium :

- Résistance d'un rivet au cisaillement : $V_{\mathrm{RS}}=$ $\alpha_{\mathrm{s}} \cdot p_{\mathrm{f}} \cdot A_{\mathrm{es}} \cdot K_{1} / \gamma_{\mathrm{m}}$

- Résistance d'un rivet à la traction : $P_{\mathrm{RT}}=$ $a \cdot p_{\mathrm{f}} \cdot A_{\mathrm{es}} / \gamma_{\mathrm{m}}$ 
Tableau 1. Séquences du processus de rivetage.

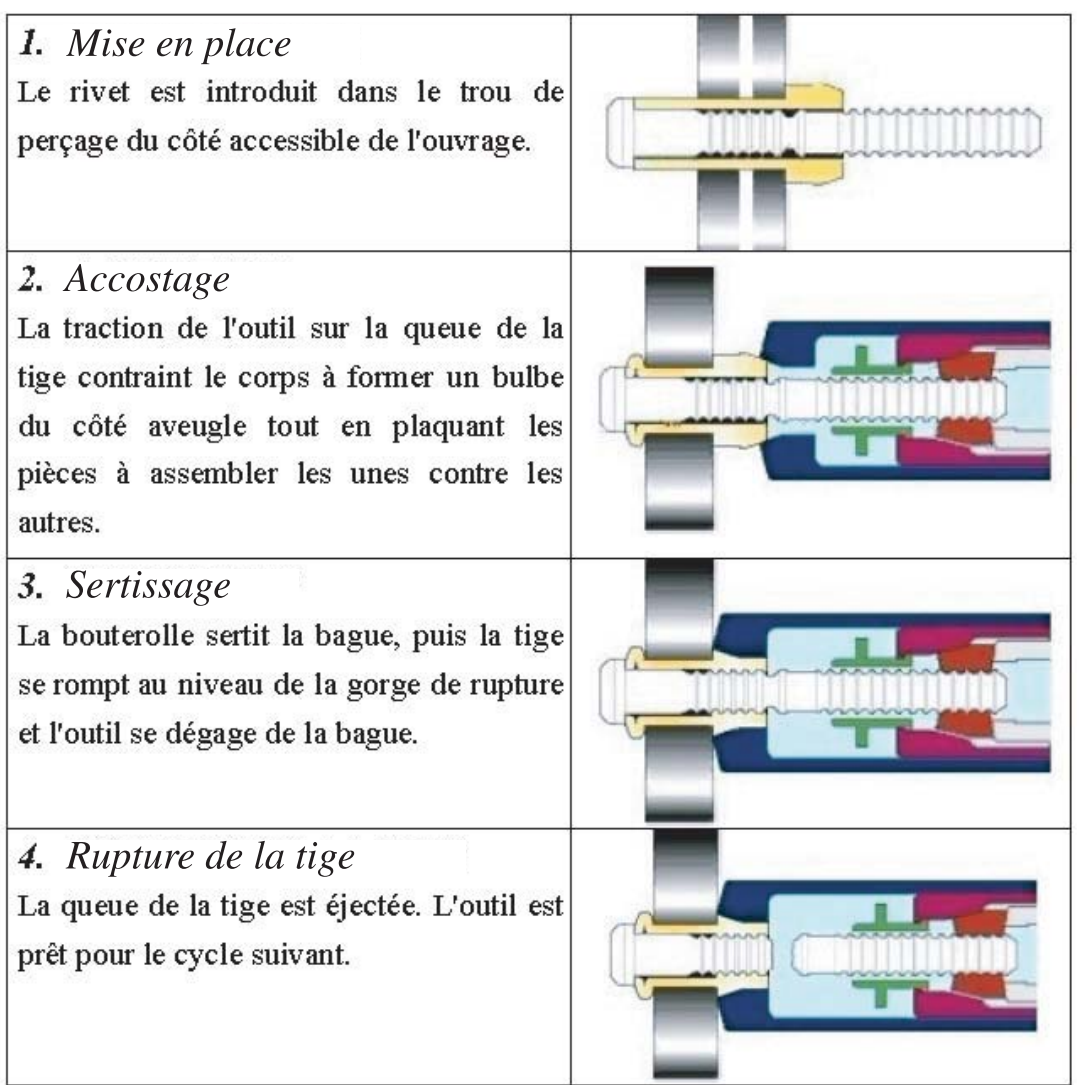

où : $\quad \alpha_{\mathrm{s}}=$ coefficient $=0,7$ pour les rivets en acier $p_{\mathrm{f}}=$ limite élastique du rivet

$A_{\text {es }}=$ aire du trou de perçage

$K_{1}=$ coefficient spécifique au rivet $=1$

$\gamma_{\mathrm{m}}=$ coefficient propre au matériau $=1,2$

$a=$ coefficient spécifique au rivet $=1$

- Dans l'Eurocode 3 [3] figurent les valeurs de résistance des rivets acier sous diverses sollicitations, dans le cas du serrage de plaques en acier :

- Résistance d'un rivet au cisaillement : $F_{\mathrm{v} . \mathrm{Rd}}=$ $0,6 . f_{\text {cis }} \cdot A_{0} / \gamma_{\mathrm{Mr}}$

- Résistance d'un rivet à la traction : $F_{\text {t.Rd }}=$ $0,6 . f_{\mathrm{tra}} \cdot A_{0} / \gamma_{\mathrm{Mr}}$

où : $\quad f_{\text {cis }}=$ résistance ultime spécifiée du rivet au cisaillement

$f_{\text {tra }}=$ résistance ultime spécifiée du rivet à la traction

$A_{0}=$ aire du trou du rivet

$\gamma_{\mathrm{Mr}}=$ coefficient de sécurité $=1,25$ pour les assemblages rivetés.

Cependant, ces normes sont généralisées à tout type de rivet, sans prise en compte de la spécificité du domaine ferroviaire (dimensions des rivets utilisés, matière, type (rivets aveugles), processus de mise en place). L'utilisation de ces modèles simplifiés conduit à des résultats erronés (assemblages trop raides et sur-dimensionnés).

Le tableau 2 présente un panel non exhaustif des rivets utilisés dans le monde industriel.

\subsection{Caractérisation du rivet seul : essais ARCAN}

Afin de dissocier le rivet de son environnement, des essais ARCAN [4] ont été réalisés sur les rivets ferroviaires utilisés. L'essai ARCAN permet de combiner et de contrôler les sollicitations de traction et de cisaillement. Deux secteurs angulaires sont liés à deux embases métalliques au milieu desquelles se trouve la liaison rivetée. L'orientation de ces secteurs par rapport à l'axe de chargement de la machine définit exactement la répartition traction/cisaillement. La variation de cette orientation permet d'obtenir plusieurs combinaisons de sollicitations. Les embases sont en MARVAL 18 traité et ont une limite élastique de $1800 \mathrm{MPa}$.

L'effort appliqué est un glisseur (pas de moment) qui passe par le centre du rivet et qui est incliné dans le plan de chargement d'un angle $\alpha$ par rapport à la normale. Les valeurs des efforts normaux et tranchants, $N$ et $T$, subis par le rivet s'expriment directement à partir de l'effort appliqué, $F$, et de l'angle d'inclinaison, $\alpha$, comme :

$$
\begin{aligned}
N(\alpha) & =F(\alpha) \cdot \cos (\alpha) \\
T(\alpha) & =F(\alpha) \cdot \sin (\alpha)
\end{aligned}
$$

Cet essai permet donc d'accéder à $n$ couples $(N(\alpha)$, $T(\alpha))$ et donc de caractériser complètement le critère de rupture à utiliser dans les simulations sans hypothèse simplificatrice ni incertitude. Il permet de découpler le rivet 
Tableau 2. Exemple de rivets utilisés dans différents domaines industriels.

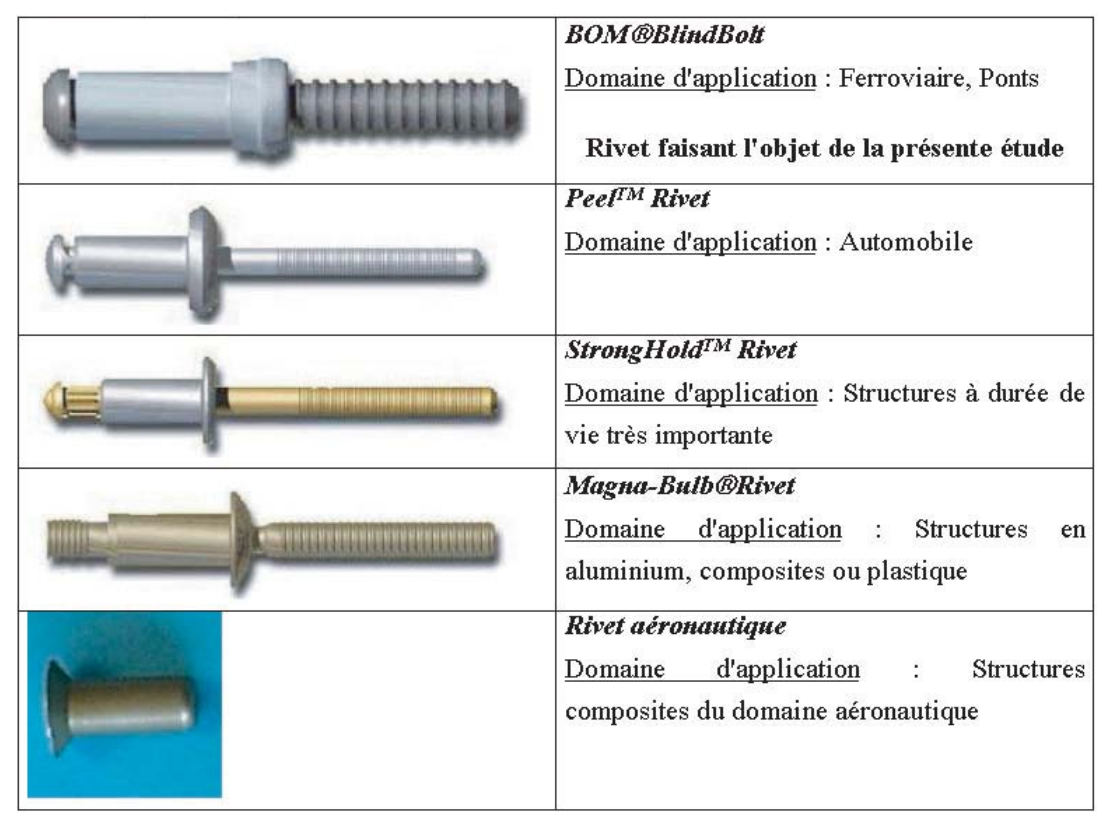

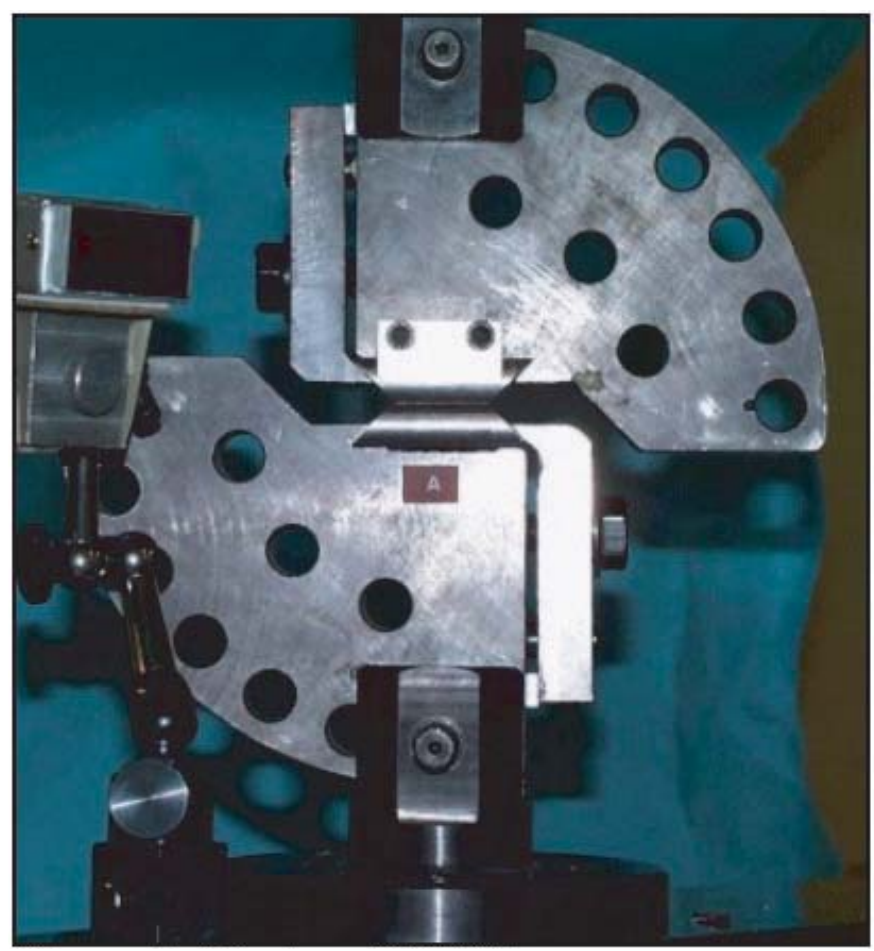

Fig. 2. Montage ARCAN.

des plaques assemblées pour accéder à une caractérisation à la rupture du rivet seul.

La figure 2 montre le montage ARCAN équipé de l'une des éprouvettes types testées.

Les essais sont menés à une vitesse de sollicitation quasi-statique $\left(V=10 \mathrm{~mm} \cdot \mathrm{min}^{-1}\right)$. Les angles d'inclinaison (exprimés en degrés) sont $\alpha=\{0,30,45,60,90\}$.
L'effort, $F$, appliqué sur l'éprouvette est mesuré par une cellule piézo-électrique Kistler.

Le tableau 3 présente les modes de rupture observés pour chaque angle de sollicitation.

Les essais ARCAN ont permis d'identifier les modes de rupture du rivet BOM-R10-8 sollicité seul (sans interaction des plaques) à différents angles. Deux éprouvettes ont été testées pour chaque angle. La figure 3 présente les réponses globales effort/déplacement pour chaque angle de sollicitation.

Les réponses globales effort/déplacement sont répétitives pour deux essais identiques réalisés. Elles montrent que plus la part de cisaillement est importante dans la sollicitation, plus l'effort de rupture croît. À $60^{\circ}$, l'effort à rupture est identique à celui enregistré à $90^{\circ}$, soit en cisaillement pur.

Un modèle numérique volumique des éprouvettes testées a été réalisé afin de simuler l'essai ARCAN réalisé (Fig. 4). Dans ce modèle, les dimensions du rivet formé numériquement présentent moins de $10 \%$ d'erreur par rapport aux données constructeur [5].

Le modèle numérique a ensuite été sollicité jusqu'à rupture du rivet pour trois des cinq angles testés $\left\{0^{\circ}, 45^{\circ}\right.$, $\left.90^{\circ}\right\}$, selon les mêmes conditions aux limites que celles des essais ARCAN. À l'aide d'un optimiseur mathématique, deux lois de comportement élasto-plastique de type puissance (du type $\sigma=\sigma_{0}+K . \varepsilon^{n}$ ) ont été identifiées. L'optimiseur mathématique utilisé permet de minimiser une fonction coût définissant le problème à résoudre à laquelle sont ajoutées des fonctions contraintes restrictives. La fonction coût représente, dans notre cas, la somme des écarts au sens des moindres carrés entre les valeurs expérimentales mesurées et celles calculées.

Aux lois identifiées ont été associés des taux de déformation limite afin de simuler la rupture du rivet lors 
Tableau 3. Modes de rupture observés à l'issue des essais ARCAN.

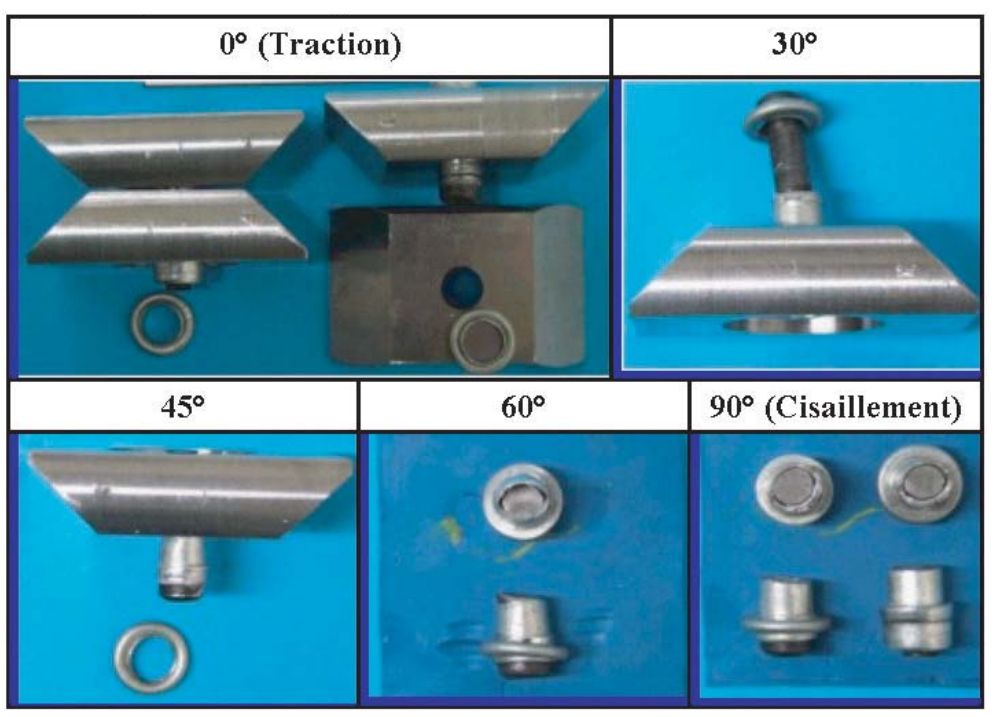

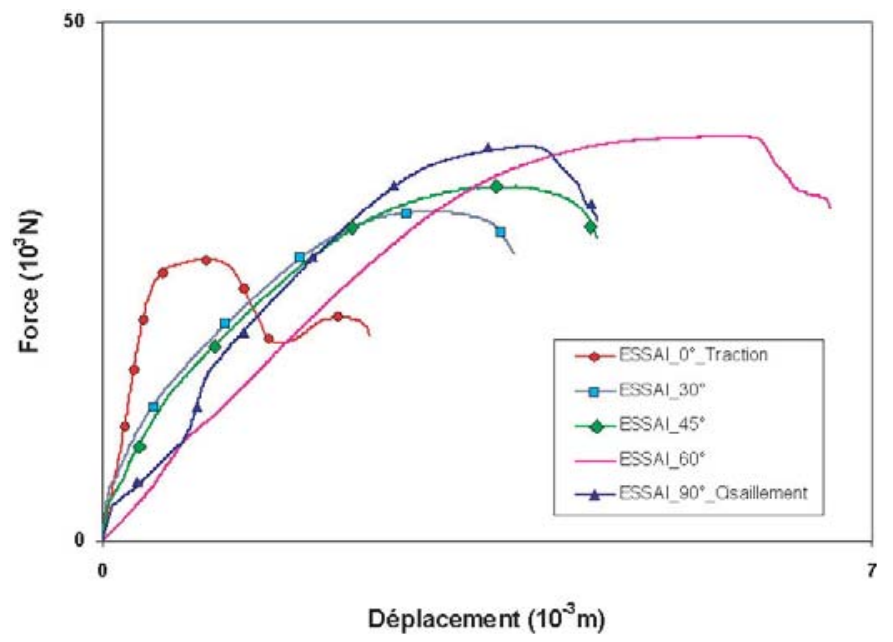

Fig. 3. Réponses globales effort/déplacement des essais ARCAN.

Tableau 4. Paramètres matériaux identifiés.

\begin{tabular}{lcc}
\hline Élément de l'assemblage & $\begin{array}{c}\text { Tige } \\
\text { du rivet }\end{array}$ & $\begin{array}{c}\text { Corps } \\
\text { du rivet }\end{array}$ \\
\hline Limite élastique du matériau $\sigma_{0}(\mathrm{MPa})$ & 950 & 365 \\
Coeff. de consistance $K(\mathrm{MPa})$ & 1171 & 522 \\
Coeff. d'écrouissage $n$ & 0,43 & 0,24 \\
Taux de déformation limite $\%$ & 81 & 114 \\
\hline
\end{tabular}

de l'essai. Ces taux correspondent au critère d'élimination des éléments volumiques lors de la simulation numérique.

Le tableau 4 récapitule les valeurs identifiées lors de l'optimisation.

Le tableau 5 présente les modes de rupture numérique obtenus avec les paramètres matériaux identifiés.

La figure 5 présente la comparaison des réponses globales effort/déplacement numériques identifiées à celles des essais réalisés.
Tableau 5. Modes de rupture obtenus après simulation.

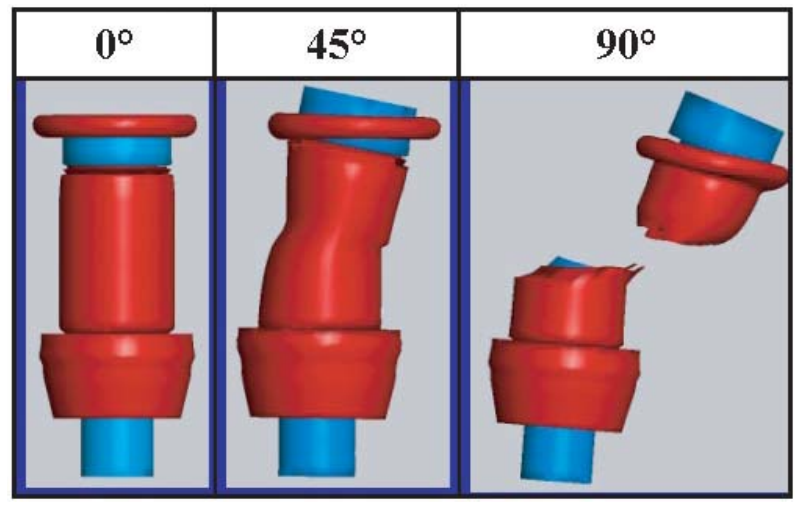

Le rivet numérique corrèle de façon satisfaisante avec les essais ARCAN réalisés, aussi bien en terme de modes de rupture que de réponses globales effort/déplacement. Connaissant le comportement du rivet seul, il est maintenant possible de le replacer dans son environnement industriel afin de caractériser globalement les liaisons rivetées, en prenant en compte l'interaction rivet/plaque.

\section{Application à une structure industrielle}

Un sous-ensemble de tramway composé de huit rivets aveugles a été sollicité en compression longitudinale quasistatique. Le but de l'essai est d'obtenir la réponse globale effort/déplacement du vérin, afin de la comparer à celle obtenue par simulation numérique. La figure 6 présente le montage d'essai. Les rivets utilisés pour cet essai sont de longueur et de diamètre différents de ceux identifiés à l'aide des essais ARCAN.

Le brancard est fixé par des étaux (aucun mouvement n'est autorisé). Un vérin manuel, d'une capacité de $250 \mathrm{kN}$, vient pincer le montant à son extrémité 


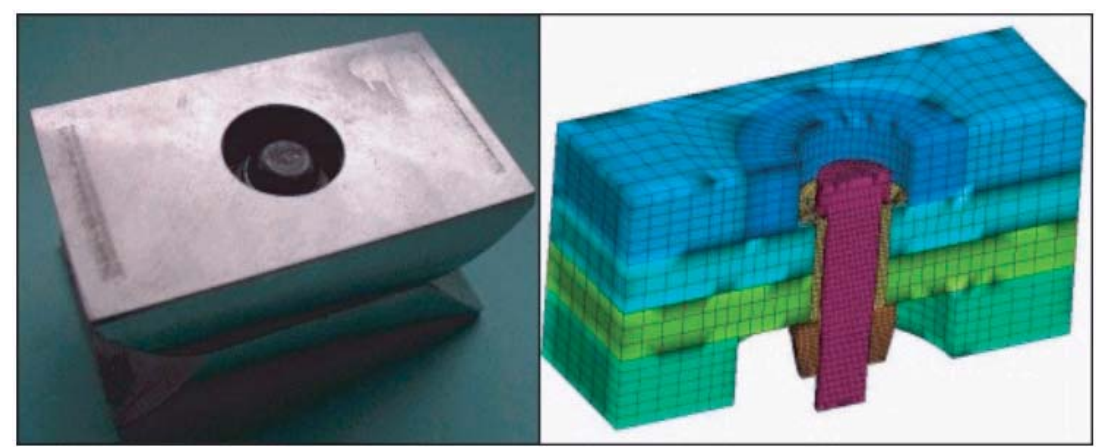

Fig. 4. Éprouvettes testée et numérique.

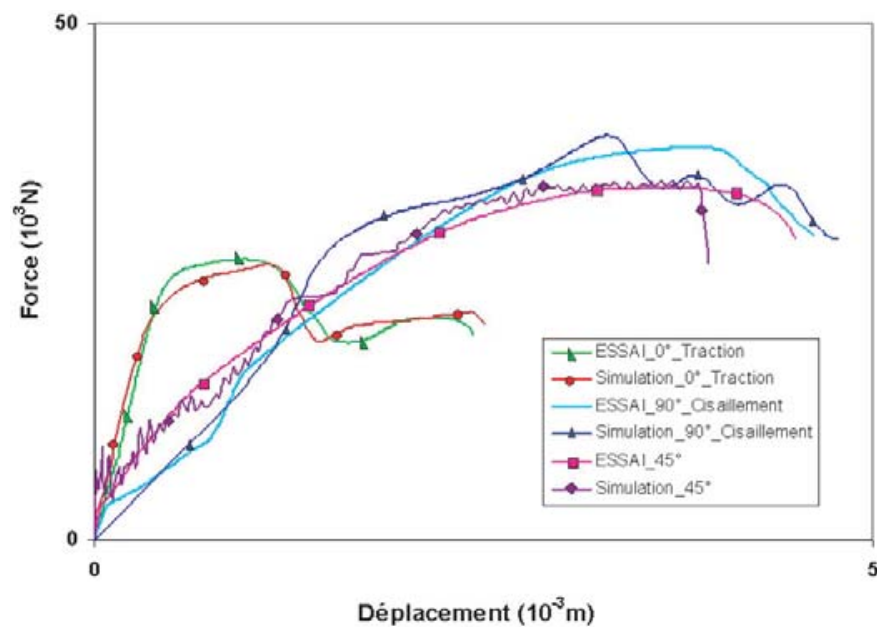

Fig. 5. Comparaison des réponses effort/déplacement numérique et expérimentale.

haute. L'effort est appliqué manuellement par palier de $2500 \mathrm{~N}$. Une mesure de déplacement est réalisée à chaque palier d'effort, à l'aide de trois comparateurs situés à différentes altitudes du montant. Le déplacement du vérin est également mesuré. Les essais sont menés jusqu'à rupture de l'assemblage.

La figure 7 présente l'assemblage après rupture des rivets ainsi que le matage constaté dans l'aluminium après désassemblage. Le matage constaté, très important, justifie la méthodologie de prise en compte de l'interaction rivet/perforation.

La modélisation de la structure testée est composée de 3477 éléments coques à trois points d'intégration et de 3444 nouds. Le comportement des rivets est mis en données par l'intermédiaire de l'interface « rivet/spotweld » du code de calcul explicite PAMCRASH ${ }^{\mathrm{TM}}$. Dans cette interface sont précisées les courbes de comportement non linéaires (en traction et cisaillement) de l'assemblage considéré, ainsi que ses valeurs de rupture associées de la liaison à prendre en compte. Le rivet y est défini par un jeu de coordonnées définissant ses extrémités. Celles-ci sont projetées sur une surface maître et une surface esclave. Les nœuds ainsi générés définissent la position réelle du rivet dans l'interface. Une relation de comportement linéaire est définie entre les déplacements et les efforts (normaux et tangentiels) pour les nouds projetés.

La figure 8 présente le modèle numérique de la structure testée ainsi que la numérotation et le positionnement des rivets.

Les courbes de comportement de l'assemblage sont issues d'un modèle numérique volumique de l'assemblage, sollicité en traction et en cisaillement (Fig. 9), avec prise en compte de l'interaction rivet/plaque. Les lois de comportement du rivet sont celles qui ont été identifiées à l'aide des essais ARCAN. Les plaques assemblées ont été caractérisées en quasi-statique à partir d'essais de traction 《 classiques ». L'assemblage est composé d'une plaque en acier S355J0 d'épaisseur $12 \mathrm{~mm}$ (côté aveugle) et d'une plaque d'alliage d'aluminium 6082 T6 d'épaisseur 8 mm (côté apparent).

Ces deux simulations (de traction et de cisaillement) permettent l'obtention des courbes de comportement de l'assemblage considéré (Fig. 10), à mettre en données dans le code de calcul explicite.

Les valeurs de rupture de l'assemblage sont pilotées par l'intermédiaire du « modèle de rupture » de l'interface « rivet/spotweld ».

Les mêmes conditions aux limites que celles de l'essai sont appliquées sur le modèle numérique. La figure 11 présente la comparaison des réponses globales effort/déplacement expérimentale et numérique.

Les courbes corrèlent de façon satisfaisante en terme de force, avec une erreur inférieure à $1 \%$. Le déplacement à rupture donné par la simulation est cependant supérieur de 1,5 mm par rapport à celui mesuré durant l'essai, soit une erreur de $4,3 \%$.

\section{Conclusion}

Une méthodologie de mise en donnée des assemblages rivetés ferroviaires est proposée. Cette méthodologie est basée sur la simulation du comportement local de l'assemblage, en prenant particulièrement en compte l'interaction entre le rivet et les plaques. Un modèle numérique volumique du rivet seul a été réalisé puis corrélé à partir d'essais ARCAN, afin d'en extraire son comportement et de le mettre en donnée dans un modèle macro-géométrique. Un sous-ensemble de structure industrielle a ensuite été 


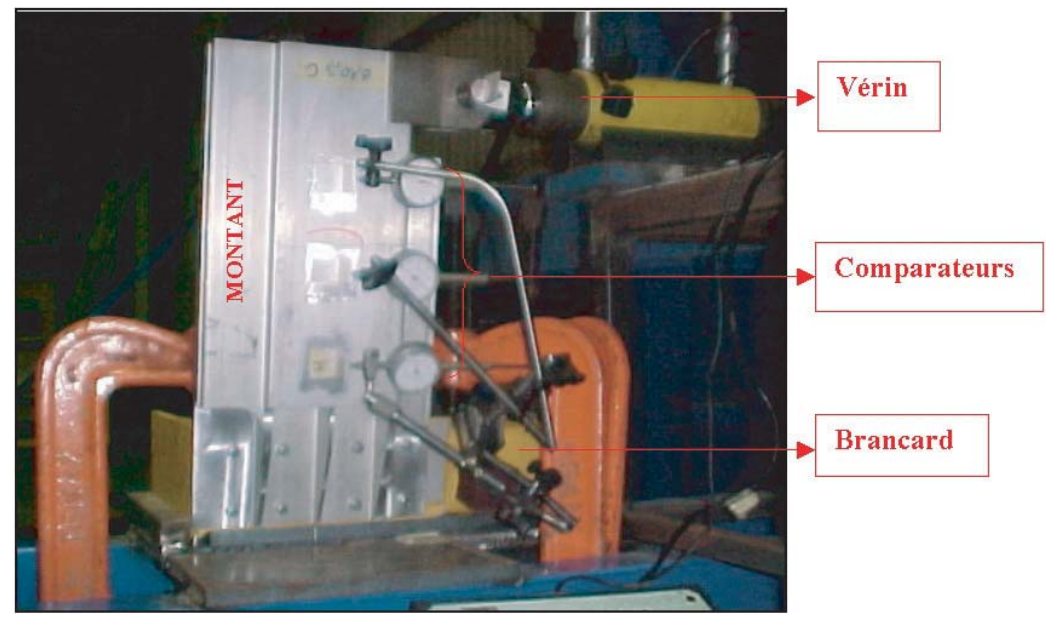

Fig. 6. Montage d'essai de compression longitudinale.

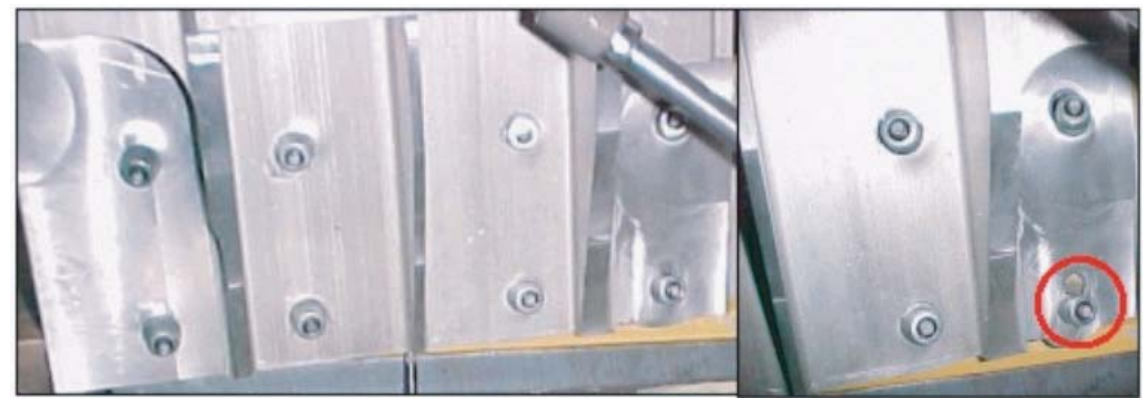

Fig. 7. État de l'assemblage après rupture et démontage.

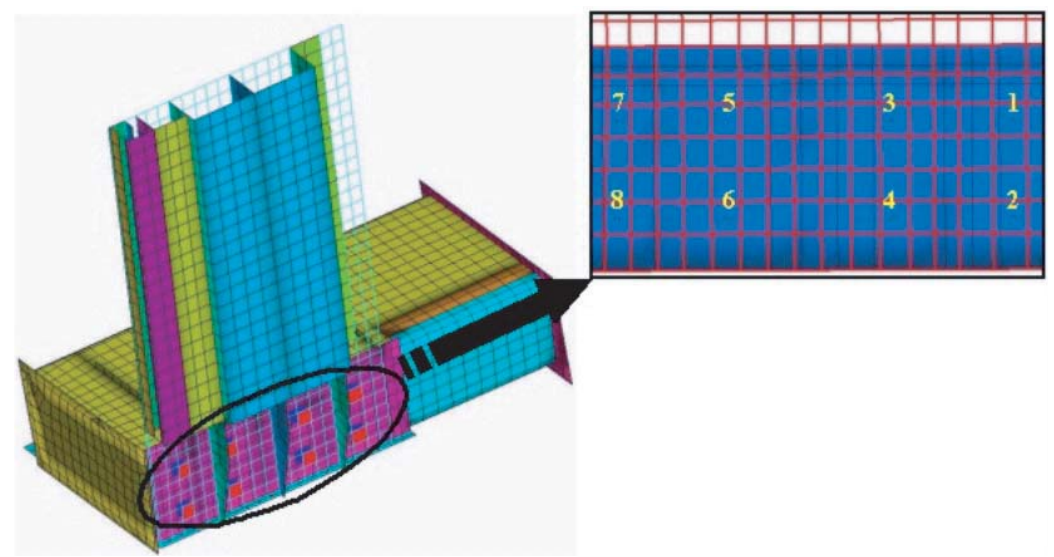

Fig. 8. Modélisation de la structure testée et positionnement des rivets.

testé en quasi-statique. Afin de prédire le comportement de cette structure, le rivet identifié précédemment a été utilisé pour obtenir numériquement les réponses globales effort/déplacement de traction et de cisaillement de l'assemblage unitaire. Ces réponses ont permis la mise en données d'une liaison macro-géométrique puis la simulation, jusqu'à rupture, de l'essai industriel réalisé. Les réponses globales effort/déplacement expérimentale et numérique corrèlent avec moins de $5 \%$ d'erreur.

La méthodologie mise en place permet d'obtenir numériquement le comportement d'un type d'assemblage donné en plaçant le rivet identifié dans l'assemblage à caractériser. Elle évite donc la mise en place de coûteuses campagnes de caractérisation et permet de dimensionner au plus juste les structures industrielles composées de rivets aveugles.

Remerciements. Les auteurs remercient vivement l'Association Nationale de la Recherche Technique qui a rendu possible ce travail de recherche dans le cadre d'une convention CIFRE, ainsi que la société ALSTOM TRANSPORT pour son support et la qualité des moyens mis à disposition pour la réalisation des essais. 


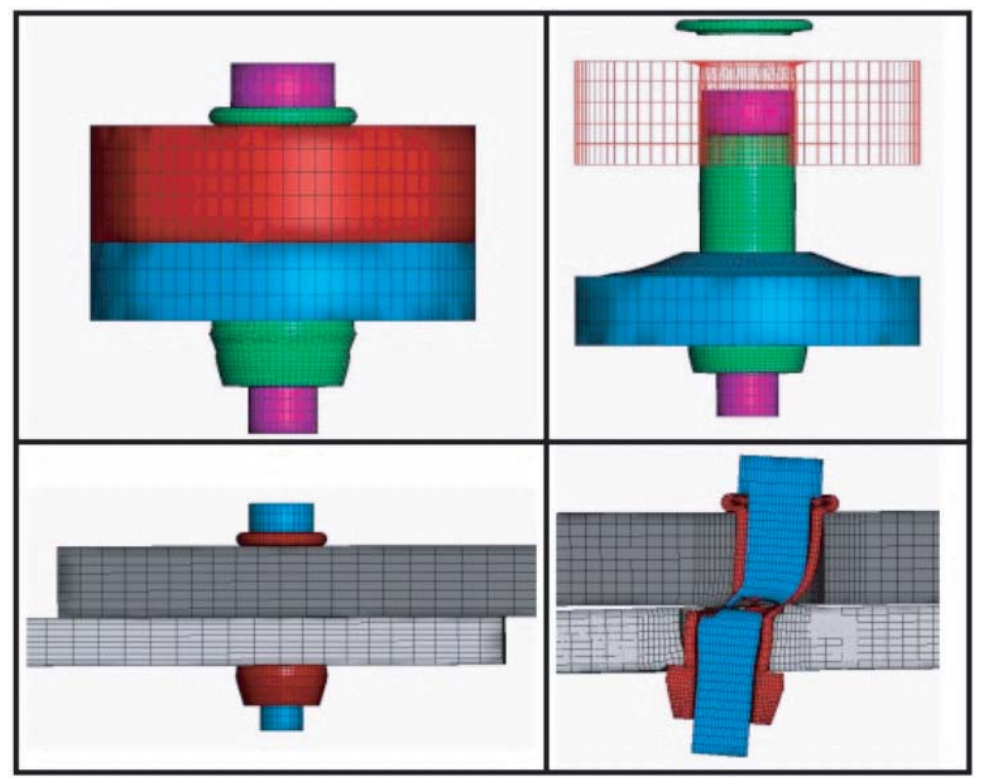

Fig. 9. Modèle volumique de l'assemblage sollicité.
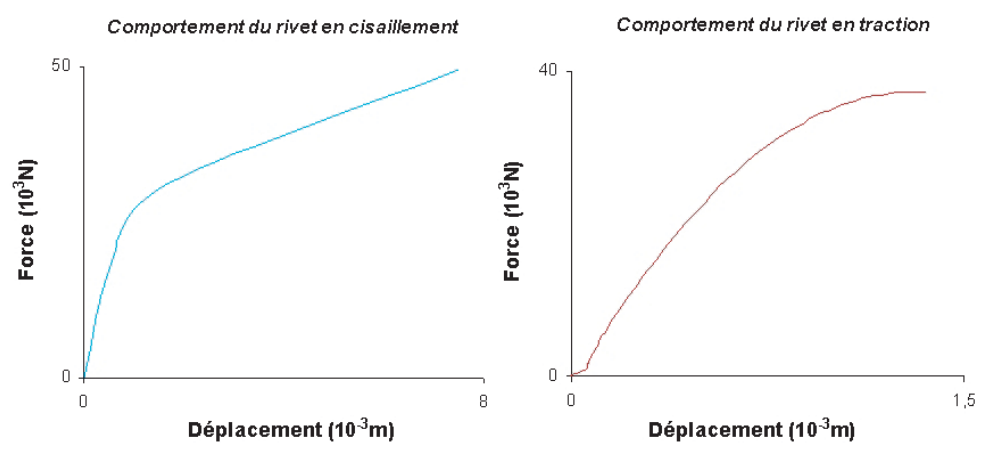

Fig. 10. Courbes de comportement d'un rivet dans l'assemblage testé.

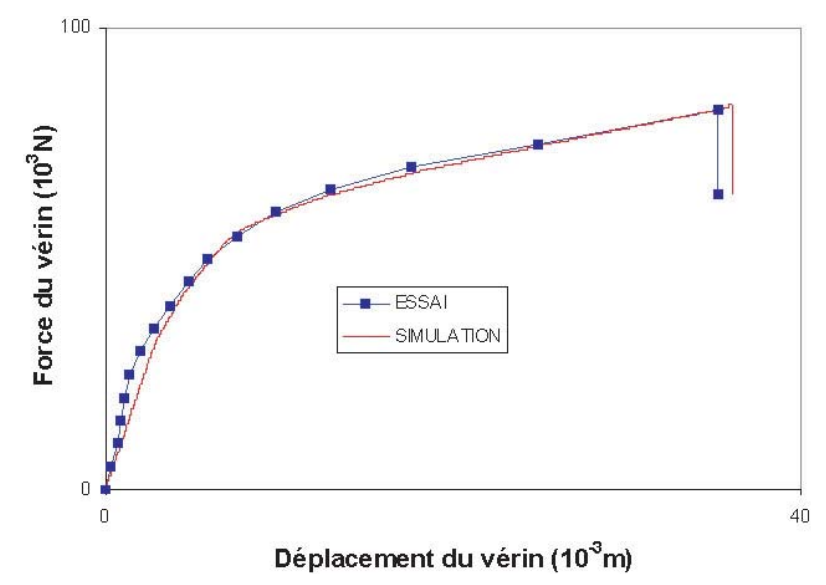

Fig. 11. Comparatif des réponses effort/déplacement numérique et expérimentale.

\section{Références}

[1] HUCK, BOM fixations aveugles, Fiche technique, 1995

[2] BRITISH STANDARD, Structural use of aluminium, Part 1 : Code of practice design, 1991

[3] EUROCODE 3 : Calcul des structures en acier, et Document d'Application Nationale, Partie 1-1 : Règles générales et règles pour les bâtiments, Chap. 6 : Assemblages sous charges statiques, AFNOR, 1992

[4] B. Langrand, Contribution à la caractérisation numérique et expérimentale d'assemblages structuraux rivetés sous sollicitation dynamique, Thèse de Doctorat, Université de Valenciennes, 1998

[5] L. Cébulski, Caractérisation numérique et expérimentale du processus de rivetage ferroviaire, Actes du XV ${ }^{\mathrm{e}}$ Congrès Français de Mécanique, 2001 